\title{
Breakdown of ergodicity in disordered $U(1)$ lattice gauge theories
}

\author{
G. Giudici, ${ }^{1,2}$ F. M. Surace, ${ }^{1,2}$ J. E. Ebot, ${ }^{1}$ A. Scardicchio, ${ }^{1,3}$ and M. Dalmonte ${ }^{1,2}$ \\ ${ }^{1}$ The Abdus Salam International Center for Theoretical Physics, Strada Costiera 11, 34151 Trieste, Italy \\ ${ }^{2}$ SISSA, Via Bonomea 265, 34136 Trieste, Italy \\ ${ }^{3}$ INFN Sezione di Trieste, Via Valerio 2, 34127 Trieste, Italy
}

(Received 17 January 2020; revised 21 May 2020; accepted 16 July 2020; published 6 August 2020)

\begin{abstract}
We show how $U(1)$ lattice gauge theories display key signatures of ergodicity breaking in the presence of a random charge background. We argue that, in such gauge theories, there is a cooperative effect of disorder and interactions in favoring ergodicity breaking: This is due to the confining nature of the Coulomb potential, which suppresses the number of available energy resonances at all distances. Such a cooperative mechanism reflects into very modest finite-volume effects: This allows us to draw a sharp boundary for the ergodic regime, and thus the breakdown of quantum chaos for sufficiently strong gauge couplings, at system sizes accessible via exact diagonalization. Our conclusions are independent on the value of a background topological angle, and are contrasted with a gauge theory with truncated Hilbert space, where instead we observe very strong finite-volume effects akin to those observed in spin chains.
\end{abstract}

DOI: 10.1103/PhysRevResearch.2.032034

Introduction. Ergodicity is one of the pillars of statistical mechanics. In the quantum regime, the ergodic hypothesis and the corresponding eigenstate thermalization hypothesis (ETH) [1,2] provide a sensible justification for the use of microcanonical and canonical ensembles in lieu of their Hamiltonian dynamics to compute long-term averages of observables. An established mechanism to circumvent thermalization is provided by Anderson localization [3]. The latter describes how noninteracting systems can feature a dynamical phase in which diffusion (and hence transport) and ergodicity are suppressed without any need to fine-tune the Hamiltonian to an integrable one. Remarkably, this mechanism has been shown to survive the introduction of interactions at the perturbative level [4,5], a phenomenon dubbed many-body localization (MBL) [6-9]. However, owing to the fundamentally more complex nature of many-body theories, establishing the breakdown of ergodicity and characterizing the ergodic/nonergodic transition in generic, interacting microscopic models has proven challenging. At the practical level, this is due to the fact that quantum chaos (which underlies ETH) is ultimately linked to the full spectral content of a theory [10], where the applicability of analytical techniques is less established with respect to low-energy studies [4,5,11-18].

An archetypal example in this field has been the onedimensional (1D) Heisenberg model with random fields [19], where, in the absence of SU(2) symmetry [20-23], the first

Published by the American Physical Society under the terms of the Creative Commons Attribution 4.0 International license. Further distribution of this work must maintain attribution to the author(s) and the published article's title, journal citation, and DOI. signatures of the breakdown of ergodicity were established at finite volume. Despite a follow-up impressive numerical effort [24-29], the precise location of the localization transition in this and similar microscopic models is still actively debated. A systematic drift of the would-be critical disorder strength was noticed already as early as in Ref. [19]. The finitesize scaling theory close to the phase transition is also still far from being satisfactory, with the numerically extracted critical exponents [26,27,30] at odds with strong disorder renormalization group predictions [31,32], and not compatible with the Harris criterion [33,34]. A recent analysis based on a different finite-size scaling ansatz was proposed where the transition point drifts linearly with system sizes [35], which, however, seems to apply, at small sizes, also to models where localization is demonstrated on solid grounds [36,37]. On top of this, a recent analysis discussed how large a system size one should analyze to go beyond the transient behavior in numerical or experimental studies [38]. The challenge is thus to identify generic mechanisms where, oppositely to the case of spin chains, interactions and disorder can cooperate (rather than compete) in establishing ergodicity breaking, potentially leading to completely novel scenarios in terms of finite-size scaling relevant to exact simulations and experiments $[39,40]$.

In this Rapid Communication, we show how lattice gauge theories (LGTs) [41,42] provide a framework within which the transition between ergodic and nonergodic behavior can be studied using conventional, well-controlled numerical methods. The key element of this observation is the cooperative effect of disorder and Coulomb law, which leads to a localization phenomenon that - as we show below-is parametrically different from what observed in other models. In concrete terms, we illustrate this mechanism in the context of the 1D lattice Schwinger model-quantum electrodynamics in 1D, illustrated schematically in Fig. 1(a). A sample of our results is depicted in Fig. 1(b), which shows the averaged ratio of 
(a)

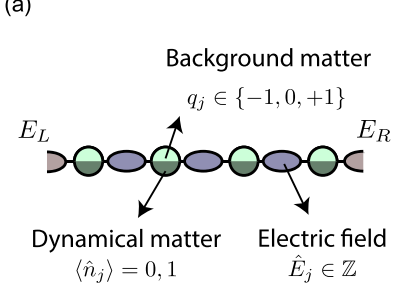

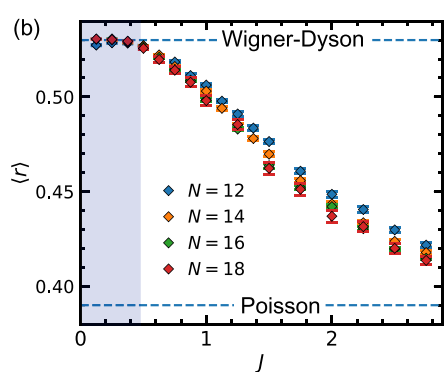

FIG. 1. (a) Schematics of $U$ (1) lattice gauge theories. The $U(1)$ gauge field lives on the links between the sites of the chain. Dynamical matter (dark green) is a fermionic variable living on the sites, while static charges (light green) are random integers which take values $0, \pm 1$. (b) Average level spacing [see Eq. (5)] as a function of the gauge coupling $J$ for different $N$ (see text). The shaded region represents the estimated ergodic phase.

nearby gaps, as a function of the gauge coupling. The results display a sharp departure from Wigner-Dyson expectations, and, crucially, the transition point from the corresponding plateau is unaffected by finite-volume effects. This behavior reflects into a modified functional form of the spectral form factor, which is not compatible with ergodicity.

Before entering into the details of our treatment, we find it useful to illustrate a qualitative reasoning why $1 \mathrm{D}$ gauge theories may be an ideal candidate to display a smoother behavior in terms of finite-volume effects, and a clearer breakdown of ergodicity. As already mentioned, contrary to typical interspin interactions, gauge-field mediated interactions typically slow down the system dynamics [43-45], and thus do not necessarily compete with disorder. In a typical BaskoAleiner-Altshuler (BAA) scenario [4], interactions open up channels for delocalization by allowing a series of local rearrangements to create a resonance between two quantum states. This leads to a competition between disorder (increasing energy differences and denominators in perturbation theory) and interactions (increasing matrix elements and therefore the numerator). In the presence of one-dimensional Coulomb law, interactions cannot be introduced perturbatively and therefore a BAA-like analysis does not work. This is because a local rearrangement of the degrees of freedom (spins or particle occupation numbers) leads to a large (even extensive) change in energy, therefore suppressing the amplitude of having a resonant process. This behavior is unrelated to the case of nonconfining long-range interactions (e.g., which decay as $1 / r^{\alpha}$, $\alpha>0$; see Refs. [46-48]), and is reflected in finite-volume properties observed in previous numerical studies [45,49,50] that focused on quench dynamics and local observables. More quantitatively, the relative strength of the interaction term with respect to the hopping term scales as $L^{-\alpha}$, hence for $\alpha>0$ the effect of disorder in the charge distribution would not be enhanced but suppressed.

Model Hamiltonian. We focus here on the 1D version of quantum electrodynamics, namely the Schwinger model [51], in its Kogut-Susskind lattice regularized version [52]. The two components of a Dirac spinor (electron and positron) sit on even and odd sites. The corresponding Hamiltonian on an open chain of $N$ sites reads

$$
\begin{aligned}
H= & -i w \sum_{n=1}^{N-1}\left(\psi_{n}^{\dagger} e^{i \varphi_{n, n+1}} \psi_{n+1}-\text { H.c. }\right) \\
& +J \sum_{n=0}^{N}\left(L_{n, n+1}+\theta / 2 \pi\right)^{2}+m \sum_{n=1}^{N}(-1)^{n} \psi_{n}^{\dagger} \psi_{n} .
\end{aligned}
$$

Matter and gauge degrees of freedom are, respectively, $N$ spinless fermions $\psi_{n}$ living on the sites and $N+1$ unbounded bosons $L_{n, n+1}$ living on the links. $L$ and $\varphi$ stand for electric field and vector potential, and they are conjugate variables: $[L, \varphi]=-i ; \theta$ is the lattice version of a topological angle that we used below to tune between confined $(\theta \neq \pi)$ and deconfined $(\theta=\pi)$ regimes [53]. Physical states $|\Psi\rangle$ in the Hilbert space satisfy Gauss law: $\left(L_{n+1, n}-L_{n, n-1}-\psi_{n}^{\dagger} \psi_{n}+\right.$ $\left.\frac{1}{2}\left[1-(-1)^{n}\right]\right)|\Psi\rangle=q_{n}|\Psi\rangle$. The $\left\{q_{n}\right\}$ are $c$ numbers representing the static charge distribution. We set $m=0$ and $w=1$ in what follows (this term is not essential for the phenomenon we describe).

Disorder-free many-body localization dynamics in this system has been reported in Ref. [45]. There, the idea was to use superselection sectors in a clean system as an effective source of correlated disorder. Other signatures of MBL in the presence of disordered on-site potentials were reported in Ref. [50]. Here, instead, we study the system properties in the presence of random, static background charges that we randomly choose in the set $q_{j}=\{0, \pm 1\}$ with equal probability.

The Hamiltonian Eq. (1) can be mapped into a spin-1/2 chain with standard techniques [54]. We define $\sigma^{\alpha}$ as the standard Pauli matrices. The resulting Hamiltonian reads

$$
H_{0}=H_{\text {Hop }}+J H_{\text {Int }}+J H_{\text {Dis }},
$$

where $H_{\text {Hop }}$ is just the hopping term $H_{\text {Hop }}=$ $-\sum_{n=1}^{N-1}\left(\sigma_{n}^{+} \sigma_{n+1}^{-}+\right.$H.c. $)$, while the second and third terms read

$$
\begin{gathered}
H_{\mathrm{Int}}=\frac{1}{2} \sum_{n=1}^{N-2} \sum_{\ell=n+1}^{N-1}(N-\ell) \sigma_{n}^{z} \sigma_{\ell}^{z}, \\
H_{\text {Dis }}=\frac{1}{2} \sum_{n=1}^{N-1}\left(\sum_{\ell=1}^{n} \sigma_{\ell}^{z}\right)\left[2 \sum_{j=1}^{n} q_{j}+\frac{(-1)^{n}-1}{2}+\frac{\theta}{\pi}\right],
\end{gathered}
$$

and describe the Coulomb interaction between dynamical charges (both terms), and the interaction between dynamical and static ones (the last term). Note that the parameter $J$ measures at the same time disorder and interaction strength. The intimate relation between these two quantities is a natural consequence of the existence of Coulomb law: In any local theory in 1D, local background charges will inevitably generate a sink (or source) of the electric field, and thus their effect on the system is tied to the gauge coupling.

Below, we consider only static charge distributions such that $\sum_{n} q_{n}=0$ and $q_{n}=0, \pm 1$. We set the left boundary electric field $L_{0,1}=0$ and restrict to charge neutrality, $\sum_{n} \psi_{n}^{\dagger} \psi_{n}=0$. In order to avoid spurious effects close to $J=0$ due to the system becoming noninteracting, we 

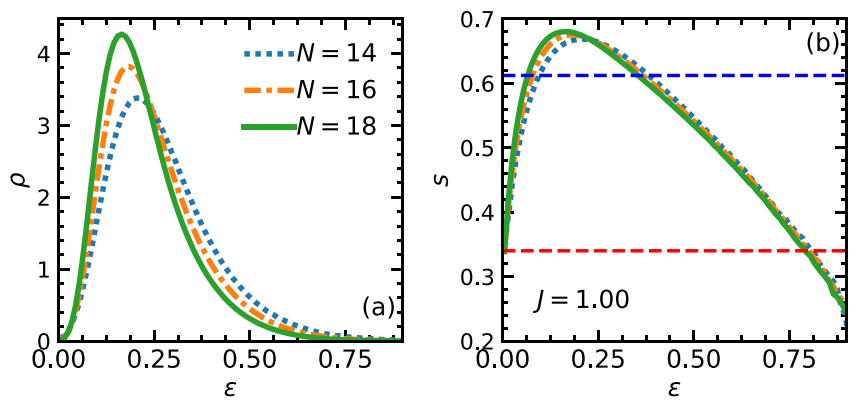

FIG. 2. (a) Spectral density and (b) entropy per site of the Hamiltonian Eq. (1) for different $N$ and $J=1$ as a function of the rescaled energy $\varepsilon=\left(E-E_{\min }\right) /\left(E_{\max }-E_{\min }\right)$. The blue dashed lines cut the spectrum keeping only the eigenvalues $E$ so that $s(E) / s_{\max }>A$. We employed $A=0.5$ for the computation of the level statistics $r$ (blue) and $A=0.9$ for the computation of the spectral form factor (red).

add a next-to-nearest-neighbor interaction of the form $H_{\epsilon}=$ $\epsilon \sum_{n=1}^{N-2} \sigma_{n}^{z} \sigma_{n+2}^{z}$ and set $\epsilon=0.5$.

Spectral diagnostics: Average level spacing ratio. To capture the breakdown of ergodicity, we focus on the spectral properties. We study the Hamiltonian in Eq. (2) by full diagonalization in the Hilbert space sector with zero total spin along $z$. In the gauge theory picture, this means zero dynamical total charge. We define the ratio between nearby gaps as

$$
r_{\alpha}=\frac{\operatorname{Min}\left\{\Delta E_{\alpha}, \Delta E_{\alpha+1}\right\}}{\operatorname{Max}\left\{\Delta E_{\alpha}, \Delta E_{\alpha+1}\right\}},
$$

where $\alpha$ labels the eigenvalues of $H$ for a given disorder realization. We average $r$ over a spectral window centered on the most likely eigenvalue, and over 1000 and 100 disorder realizations for $N<18$ and $N=18$, respectively.

As illustrated in Fig. 2(a), the Coulomb interaction makes the eigenvalue distribution $\rho$ strongly asymmetric, due to the superlinear scaling of the largest eigenvalues in the spectrum. We thus cut the tails of the spectral density $\rho$ by monitoring the thermodynamic entropy per site: $s=\log \rho / L$. To compute the level statistics $r$ we keep only the eigenvalues $E$ for which $s(E) / s_{\max }>0.9$ [blue dashed line in Fig. 2(b)]. This is illustrated in Fig. 3(a), where the energy-resolved $r$ value is plotted as a function of gauge coupling and energy

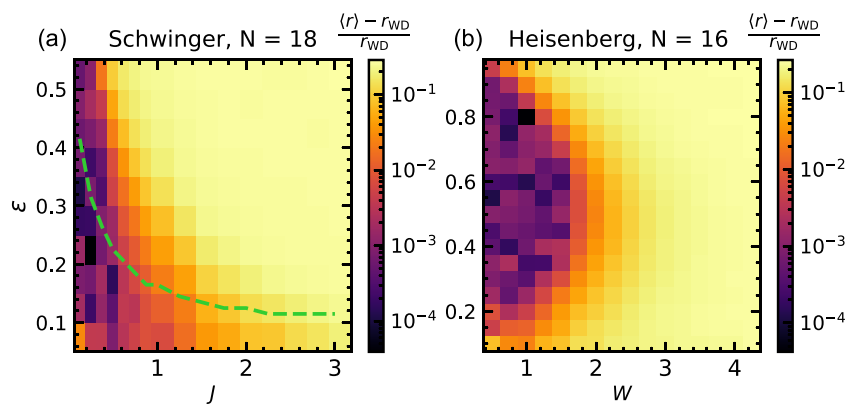

FIG. 3. Energy-resolved $r$ as a function of the rescaled energy $\varepsilon$, and (a) gauge coupling $J$ in the lattice gauge theory and (b) disorder strength $W$ in the Heisenberg model. The green dashed line indicates the position of the maximum of the spectral density. density [55]. Considering the full spectrum does not lead to quantitative changes in the transition region.

The resulting scaling of $r$ vs $J$ is plotted in Fig. 1. The results illustrate how compatibility with a Wigner-Dyson distribution of the energy levels breaks down at around $J \simeq 0.5$; contrary to the Heisenberg model case (where the critical disorder strength increases by $50 \%$ when comparing $N=12$ and $N=18$ ), there is no appreciable finite-volume drift. We note that this behavior is fully compatible with the energy-resolved pattern of $r$ plotted in Fig. 3(a): Indeed, only states very close to the ground state are not localized, and as such, the global value of $r$ is dominated by the vast majority of states that is localized [note that the vertical axis in Fig. 3(a) is limited to $\varepsilon \in[0.05,0.55]$ for the sake of clarity]. The ergodic region (shaded) is followed by a regime where $\langle r\rangle$ takes intermediate values: While it is not possible to reliably distinguish between emergent integrability (denoted by Poisson statistics) and an intermediate value of $r$, there is a clear finite-size trend toward the former for $J>1$. Within statistical errors, we do not observe a clear crossing: Longer chains routinely have smaller $r$ values with respect to shorter chains. Finally, let us note that our diagnostics may actually underestimate the extent of the nonergodic regime, as there exist random-matrix models [56] where ergodicity is broken even in regimes where $r$ is compatible with a Gaussian orthogonal ensemble (GOE).

Spectral diagnostics: Form factor. As further evidence of the breakdown of ergodicity, we analyze spectral correlations which go beyond nearby eigenvalues via the spectral form factor (SFF), defined as

$$
K(\tau)=\frac{1}{\mathcal{Z}}\left|\sum_{\alpha} g\left(\tilde{E}_{\alpha}\right) e^{i 2 \pi \tau \tilde{E}_{\alpha}}\right|^{2},
$$

where $\tilde{E}_{\alpha}$ are the unfolded eigenvalues. In order to smooth the effects due to boundaries of the spectrum, we apply a Gaussian filter $g(x)=e^{-\frac{(x-\mu)^{2}}{2(\eta \sigma)^{2}}}$, with $\mu$ and $\sigma$ the average and variance of the disorder realization of the unfolded spectrum. $\eta$ quantifies the strength of the filter, and we take $\eta=0.3$ in what follows. $\mathcal{Z}=\sum_{\alpha}\left|g\left(\tilde{E}_{\alpha}\right)\right|^{2}$ is a normalization so that $K(\tau) \simeq 1$ for large $\tau$. Before applying the filter, we cut the edges of the spectrum according to $s(E) / s_{\max }>0.5$, which means we take a fraction of eigenvalues larger than 0.9 . Upon unfolding, the Heisenberg time $t_{\mathrm{H}}$, corresponding to the timescale beyond which the discrete nature of the spectrum manifests itself and thus nonuniversal features kick in, is set to unity. The SFF in Eq. (6) is computed for each disorder realization for $\tau \in[0,1]$ and an average over disorder is performed for each value of $\tau$.

The analysis of $K(\tau)$ allows one to probe if the system is ergodic $[35,36,57]$. This can be done by comparing the averaged SFF with the SFF expected from an ensemble of orthogonal random matrices with Gaussian entries (GOE), $K_{\mathrm{GOE}}=2 \tau-\tau \log (1+2 \tau)$. We call $t_{\mathrm{GOE}}$ the time at which the averaged SFF approaches the GOE prediction. If the system is ergodic [36], this corresponds to the Thouless time, and one has $t_{\mathrm{GOE}} / t_{\mathrm{H}} \rightarrow 0$ in the thermodynamic limit (specifically, the Thouless time shall increase algebraically with $N$ ).

In Figs. 4(a) and 4(b), we plot the spectral form factor in the Schwinger model and Heisenberg model in their ergodic 

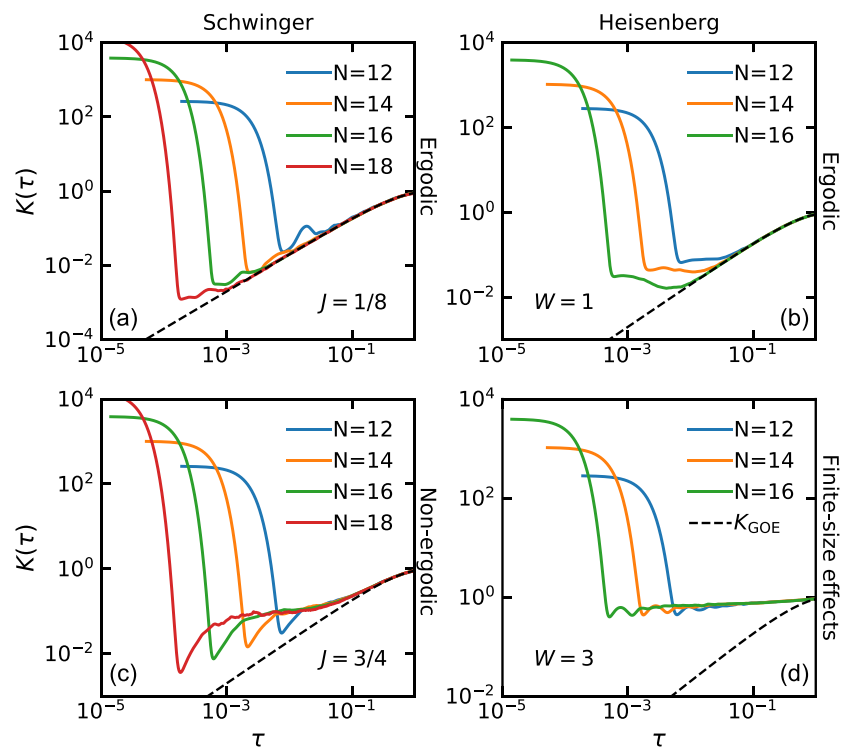

FIG. 4. Comparison between (a), (c) the SFF of the Hamiltonian Eq. (2) and (b), (d) of the Heisenberg model with on-site disorder. (a), (b) In the deep ergodic region the SFF approaches the GOE prediction at times which decrease exponentially with the size of the system. (c) For $J=3 / 4$ in the LGT the bulk of the spectrum is nonergodic and the SFF deviates from the GOE prediction at intermediate times. (d) For $W=3$ in the Heisenberg chain the level statistics is still flowing to Wigner-Dyson (WD), however, the small effective localization length prevents accessing ergodic properties of the thermodynamic limit.

regions: In both cases, the Thouless time is clearly decreasing with system size, further confirming the ergodic nature of the phase. The results in Fig. 4(c) correspond to a regime of gauge couplings whose $r$ value departs from GOE: Such a departure is indeed confirmed by the fact that the $t_{\mathrm{GOE}} / t_{\mathrm{H}}$ is not decreasing with system size, and oppositely, the SFF seems to collapse on a finite linear region, which implies $\ln t_{\mathrm{GOE}} \sim N$; this timescale directly indicates that the system is not ergodic, and it is suggestive of an emergent localization even at this value of the coupling. We note that, in this parameter regime, we do not observe saturation of the Thouless time, which is instead evident in spin models [see Fig. 4(d) and Ref. [35]].

Finally, we comment on the consequences of our numerical observations on transport properties. In the region $J<0.5$, our numerical data are consistent with a power-law scaling. Transport properties in this regime are thus expected to be qualitatively similar to the case of the Heisenberg model in random fields - see Ref. [54]. Oppositely, for larger values of the disorder, the fact that the ratio $t_{\mathrm{GOE}} / t_{\mathrm{H}}$ is size independent suggests that the suppression of transport is related to a sizeindependent scale, a very different scenario with respect to what is observed in Heisenberg models, characterized instead by anomalous transport properties [58-61]: While it is not possible to immediately connect this mechanism to confinement, we naturally expect this emergent scale to be connected with the string tension, as the latter is size independent and is the only parameter needed to characterize Coulomb interactions at large scales.
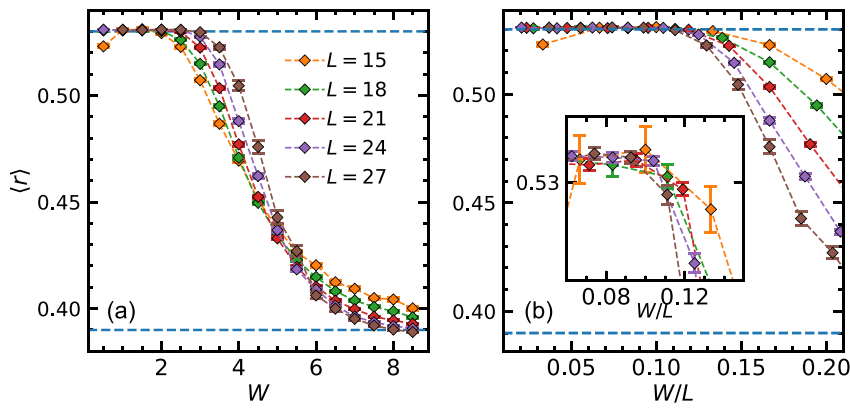

FIG. 5. Average level spacing ratio for the constrained spin model $H_{\mathrm{QLM}}$, corresponding to the Schwinger model with truncated gauge fields. The finite-size scaling of $\langle r\rangle$ exhibits the same phenomenology as in the Heisenberg chain: (a) $\langle r\rangle$ vs $W$ shows a crossing point drifting on the right for increasing $N$, and (b) $\langle r\rangle$ vs $W / N$ gives a good data collapse for $W / N<0.1$.

Origin of ergodicity breaking. We conjecture that the origin of ergodicity breaking in lattice gauge theories stems from the fact that Coulomb law-which is acting at all energy scalesfurther constrains the system dynamics, and thus acts as an amplifier of any background disorder. In fact, for increasing system sizes, a larger fraction of the states of the spectrum will feature regions with a large accumulation of charge: As a consequence, the electrostatic energy (which is locally unbounded) becomes dominant and the effect of Coulomb interactions is enhanced. The presence of an unbounded energy density, which contrasts with the usual behavior of spin models, does not affect low-energy states, but has important consequences on the rest of the spectrum: For instance, it systematically reduces the number of available resonances when size is increased. In order to substantiate this statement, we studied (1) the Schwinger model in its deconfined regime, $\theta=\pi$, and (2) a quantum link version of the model with truncated gauge fields, where Coulomb law is washed out by the truncation. We note that the fact that (de)confinement is not crucial here is not unexpected, as the latter is a phenomenon that only dictates the dynamics in the vicinity of the vacuum state. See Ref. [54] for an analysis of the effect of the topological angle in the Schwinger model.

In Figs. 5(a) and 5(b), we instead show $r$ versus the disorder strength $W$ in a quantum link model (QLM) in the presence of a background disorder (see Ref. [54] for details on the model) [62]. In the presence of strong nearest-neighbor interactions, the system dynamics can be mapped exactly [54,63] into a constrained spin model of the form $H_{\mathrm{QLM}}=$ $\sum_{i}\left(W_{i} n_{i}-\sigma_{i}^{x}\right), n_{i} n_{i+1}=n_{i} n_{i+2}=0$, where $n=\left(1-\sigma^{z}\right) / 2$. We attempted a collapse scaling following [26], and assuming a finite transition point $W_{c}$ and correlation length critical exponent $v$. The best fitting $W_{c}$ and $v$ seem to increase linearly with size. The scaling of $r$ follows rather closely the functional form proposed in Ref. [35]. These two observations indicate that, even in this model, the available system sizes are not sufficient to determine whether ergodicity is broken in the thermodynamic limit [38]. Overall, the findings on these two models support our conjecture above.

Conclusions and outlook. We have provided numerical evidence for the breakdown of ergodicity in disordered $U(1)$ lattice gauge theories. Our results do not immediately indicate 
if localization kicks in right after such a breakdown, or if an intermediate nonergodic, delocalized regime occurs. Further studies based on localization-specific diagnostics and transport properties may elucidate this aspect. The dynamical consequences of our results are immediately testable on quantum simulation platforms, where the many-body dynamics of $U(1)$ lattice gauge theories has been recently realized [63-65], and, based on the nature of the interactions, might be extended to Yang-Mills theories.
Acknowledgments. We thank D. Abanin, M. Amini, J. Chalker, M. Heyl, V. Kravtsov, D. Luitz, F. Pollmann, T. Prosen, and J. Zakrzewski for discussions. This work is partly supported by the ERC under Grant No. 758329 (AGEnTh), by the Quantera programme QTFLAG, and has received funding from the European Union's Horizon 2020 research and innovation programme under Grant Agreement No 817482. This work has been carried out within the activities of TQT.
[1] M. Srednicki, Phys. Rev. E 50, 888 (1994).

[2] J. M. Deutsch, Phys. Rev. A 43, 2046 (1991).

[3] P. W. Anderson, Phys. Rev. 109, 1492 (1958).

[4] D. M. Basko, I. L. Aleiner, and B. L. Altshuler, Ann. Phys. 321, 1126 (2006).

[5] I. V. Gornyi, A. D. Mirlin, and D. G. Polyakov, Phys. Rev. Lett. 95, 206603 (2005).

[6] E. Altman and R. Vosk, Annu. Rev. Condens. Matter Phys. 6, 383 (2015).

[7] R. Nandkishore and D. A. Huse, Annu. Rev. Condens. Matter Phys. 6, 15 (2015).

[8] J. Z. Imbrie, V. Ros, and A. Scardicchio, Ann. Phys. 529, 1600278 (2017).

[9] D. A. Abanin, E. Altman, I. Bloch, and M. Serbyn, Rev. Mod. Phys. 91, 021001 (2019).

[10] F. Haake, in Quantum Coherence in Mesoscopic Systems, edited by B. Kramer, NATO ASI Series (Series B: Physics), Vol. 254 (Springer, Berlin, 1991), pp. 583-595.

[11] L. Fleishman and P. W. Anderson, Phys. Rev. B 21, 2366 (1980).

[12] B. L. Altshuler, Y. Gefen, A. Kamenev, and L. S. Levitov, Phys. Rev. Lett. 78, 2803 (1997).

[13] J. Z. Imbrie, J. Stat. Phys. 163, 998 (2016).

[14] V. Ros, M. Müller, and A. Scardicchio, Nucl. Phys. B 891, 420 (2015).

[15] W. De Roeck and F. Huveneers, Commun. Math. Phys. 332, 1017 (2014).

[16] W. De Roeck and F. Huveneers, Phys. Rev. B 90, 165137 (2014).

[17] W. De Roeck, F. Huveneers, M. Müller, and M. Schiulaz, Phys. Rev. B 93, 014203 (2016).

[18] A. Chandran, A. Pal, C. R. Laumann, and A. Scardicchio, Phys. Rev. B 94, 144203 (2016).

[19] A. Pal and D. A. Huse, Phys. Rev. B 82, 174411 (2010).

[20] P. Prelovšek, O. S. Barišić, and M. Žnidarič, Phys. Rev. B 94, 241104(R) (2016).

[21] A. C. Potter and R. Vasseur, Phys. Rev. B 94, 224206 (2016).

[22] I. V. Protopopov, W. W. Ho, and D. A. Abanin, Phys. Rev. B 96, 041122(R) (2017).

[23] I. V. Protopopov, R. K. Panda, T. Parolini, A. Scardicchio, E. Demler, and D. A. Abanin, Phys. Rev. X 10, 011025 (2020).

[24] A. De Luca and A. Scardicchio, Europhys. Lett. 101, 37003 (2013).

[25] J. Goold, C. Gogolin, S. R. Clark, J. Eisert, A. Scardicchio, and A. Silva, Phys. Rev. B 92, 180202(R) (2015).
[26] D. J. Luitz, N. Laflorencie, and F. Alet, Phys. Rev. B 91, 081103(R) (2015).

[27] F. Pietracaprina, G. Parisi, A. Mariano, S. Pascazio, and A. Scardicchio, J. Stat. Mech. (2017) 113102.

[28] M. Žnidarič, A. Scardicchio, and V. K. Varma, Phys. Rev. Lett. 117, 040601 (2016).

[29] F. Alet and N. Laflorencie, C. R. Phys. 19, 498 (2018).

[30] N. Macé, F. Alet, and N. Laflorencie, Phys. Rev. Lett. 123, 180601 (2019).

[31] R. Vosk, D. A. Huse, and E. Altman, Phys. Rev. X 5, 031032 (2015).

[32] P. T. Dumitrescu, A. Goremykina, S. A. Parameswaran, M. Serbyn, and R. Vasseur, Phys. Rev. B 99, 094205 (2019).

[33] A. Chandran, C. R. Laumann, and V. Oganesyan, arXiv: 1509.04285 .

[34] A. B. Harris, J. Phys. C: Solid State Phys. 7, 1671 (1974).

[35] J. Šuntajs, J. Bonča, T. Prosen, and L. Vidmar, arXiv:1905.06345.

[36] P. Sierant, D. Delande, and J. Zakrzewski, Phys. Rev. Lett. 124, 186601 (2020).

[37] D. A. Abanin, J. H. Bardarson, G. D. Tomasi, S. Gopalakrishnan, V. Khemani, S. A. Parameswaran, F. Pollmann, A. C. Potter, M. Serbyn, and R. Vasseur, arXiv:1911.04501.

[38] R. K. Panda, A. Scardicchio, M. Schulz, S. R. Taylor, and M. Žnidarič, Europhys. Lett. 128, 67003 (2019).

[39] M. Schreiber, S. S. Hodgman, P. Bordia, H. P. Lüschen, M. H. Fischer, R. Vosk, E. Altman, U. Schneider, and I. Bloch, Science 349, 842 (2015).

[40] J. Smith, A. Lee, P. Richerme, B. Neyenhuis, P. W. Hess, P. Hauke, M. Heyl, D. A. Huse, and C. Monroe, Nat. Phys. 12, 907 (2016).

[41] K. G. Wilson, Phys. Rev. D 10, 2445 (1974).

[42] I. Montvay and G. Muenster, Quantum Fields on a Lattice (Cambridge University Press, Cambridge, UK, 1994).

[43] S. Kühn, E. Zohar, J. I. Cirac, and M. C. Bañuls, J. High Energy Phys. 07 (2015) 130.

[44] T. Pichler, M. Dalmonte, E. Rico, P. Zoller, and S. Montangero, Phys. Rev. X 6, 011023 (2016).

[45] M. Brenes, M. Dalmonte, M. Heyl, and A. Scardicchio, Phys. Rev. Lett. 120, 030601 (2018).

[46] A. L. Burin, arXiv:cond-mat/0611387.

[47] N. Y. Yao, C. R. Laumann, S. Gopalakrishnan, M. Knap, M. Müller, E. A. Demler, and M. D. Lukin, Phys. Rev. Lett. 113, 243002 (2014).

[48] A. L. Burin, Phys. Rev. B 92, 104428 (2015). 
[49] R. M. Nandkishore and S. L. Sondhi, Phys. Rev. X 7, 041021 (2017).

[50] A. A. Akhtar, R. M. Nandkishore, and S. L. Sondhi, Phys. Rev. B 98, 115109 (2018).

[51] J. Schwinger, Phys. Rev. 82, 914 (1951).

[52] J. Kogut and L. Susskind, Phys. Rev. D 11, 395 (1975).

[53] S. Coleman, Ann. Phys. 101, 239 (1976).

[54] See Supplemental Material at http://link.aps.org/supplemental/ 10.1103/PhysRevResearch.2.032034 for (i) the details on the Schwinger Model and the Quantum Link Model and the corresponding mappings to quantum spin chains, and for (ii) additional results on the scaling on $t_{\mathrm{GOE}}$ and the spectral statistics for non-zero topological angle.

[55] Similar behavior occurs in the Bose-Hubbard model [66].

[56] V. E. Kravtsov, I. M. Khaymovich, E. Cuevas, and M. Amini, New J. Phys. 17, 122002 (2015).

[57] M. Serbyn, Z. Papić, and D. A. Abanin, Phys. Rev. B 96, 104201 (2017).
[58] K. Agarwal, S. Gopalakrishnan, M. Knap, M. Müller, and E. Demler, Phys. Rev. Lett. 114, 160401 (2015).

[59] D. J. Luitz and Y. Bar Lev, Phys. Rev. Lett. 117, 170404 (2016).

[60] D. J. Luitz and Y. B. Lev, Ann. Phys. 529, 1600350 (2017).

[61] D. J. Luitz, I. M. Khaymovich, and Y. B. Lev, SciPost Phys. Core 2, 006 (2020).

[62] D. Banerjee, M. Dalmonte, M. Müller, E. Rico, P. Stebler, U.-J. Wiese, and P. Zoller, Phys. Rev. Lett. 109, 175302 (2012).

[63] F. M. Surace, P. P. Mazza, G. Giudici, A. Lerose, A. Gambassi, and M. Dalmonte, Phys. Rev. X 10, 021041 (2020).

[64] E. A. Martinez, C. A. Muschik, P. Schindler, D. Nigg, A. Erhard, M. Heyl, P. Hauke, M. Dalmonte, T. Monz, P. Zoller et al., Nature (London) 534, 516 (2016).

[65] H. Bernien, S. Schwartz, A. Keesling, H. Levine, A. Omran, H. Pichler, S. Choi, A. S. Zibrov, M. Endres, M. Greiner et al., Nature (London) 551, 579 (2017).

[66] P. Sierant and J. Zakrzewski, New J. Phys. 20, 043032 (2018). 